\title{
Long-Term Changes in Self-Report Auditory Verbal Hallucinations in Patients with Schizophrenia Using Clozapine
}

\author{
In Won Chung ${ }^{1,2}$, Seong Hoon Jeong ${ }^{3}$, Hee Yeon Jung ${ }^{4,5}$, Tak Youn ${ }^{1,2}$, Se Hyun Kim ${ }^{6,7}$, and Yong Sik Kim ${ }^{1,2 \bowtie}$ \\ ${ }^{1}$ Department of Psychiatry, Dongguk University International Hospital, Goyang, Republic of Korea \\ ${ }^{2}$ Institute of Clinical Psychopharmacology, Dongguk University College of Medicine, Goyang, Republic of Korea \\ ${ }^{3}$ Department of Psychiatry, Daejeon Eulji University Hospital, Daejeon, Republic of Korea \\ ${ }^{4}$ Department of Psychiatry, SMG-SNU Boramae Medical Center, Seoul, Republic of Korea \\ ${ }^{5}$ Department of Psychiatry and Behavioral Science and Institute of Human Behavioral Medicine, Seoul National University College of Medicine, \\ Seoul, Republic of Korea \\ ${ }^{6}$ Department of Psychiatry, Seoul National University Hospital, Seoul National University College of Medicine, Seoul, Republic of Korea \\ ${ }^{7}$ Institute of Human Behavioral Medicine, Seoul National University College of Medicine, Seoul, Republic of Korea
}

This study explored long-term changes in self-report auditory verbal hallucinations (AVHs) among patients with schizophrenia taking clozapine. Forty-four patients who were evaluated more than twice and were above the mild severity category on the Hamilton Program for Schizophrenia Voices Questionnaire (HPSVQ) were enrolled. The mean observation period was 492.5 \pm 350.1 days (median, 452 days). The mean total, physical, and emotional factor scores on the HPSVQ were significantly reduced from baseline to the final observations except for one item "interference with life", which was not significantly reduced. Regarding the time-dependent longitudinal changes modeled using linear mixed-effect regression, the total and physical factor scores showed significant changes during the first year, but the emotional factor score did not satisfy a more stringent level of significance. Female gender was negatively associated with the reduction in total and physical factor scores. The duration of treatment with clozapine also had a negative relationship with the reductions in all three scores.

Psychiatry Investig 2019;16(5):403-406

Key Words Auditory verbal hallucinations, Clozapine, HPSVQ, Schizophrenia, Self-report.

\section{INTRODUCTION}

Auditory verbal hallucinations (AVHs) that are the most characteristic symptom of schizophrenia, ${ }^{1-3}$ have multidimensional aspects that are mostly assessed with interviewer-rated scales, such as the Psychotic Symptom Rating Scales-Auditory Hallucination Subscale (PSYRATS-AHS). ${ }^{4,5}$ However, quantitative indicators of PSYRATS-AHS do not correlate well with the subjective distress. ${ }^{4,6,7}$ Studies indicate that patients can report AVHs reliably, ${ }^{8-10}$ and self-reported outcomes can inform clinical practice. ${ }^{11,12}$ The Hamilton Program for Schizophrenia Voices Questionnaire (HPSVQ) is a self-report scale

Received: December 4, 2018 Revised: March 19, 2019

Accepted: March 20, 2019

$\triangle$ Correspondence: Yong Sik Kim, MD, PhD

Department of Psychiatry, Dongguk University International Hospital, Institute of Clinical Psychopharmacology, 27 Dongguk-ro, Ilsandong-gu, Goyang 10326, Republic of Korea

Tel: +82-31-961-7239, Fax: +82-31-961-7236, E-mail: kys@snu.ac.kr

(a) This is an Open Access article distributed under the terms of the Creative Commons Attribution Non-Commercial License (https://creativecommons.org/licenses/bync/4.0) which permits unrestricted non-commercial use, distribution, and reproduction in any medium, provided the original work is properly cited. that includes domains similar to those of PSYRATS-AHS. ${ }^{78,13}$ HPSVQ is characterized by good psychometric properties and sensitive enough to changes in AVHs. ${ }^{8}$

Though clozapine is highly effective for reducing AVHs, ${ }^{4}$ more than $25 \%$ of patients still suffer from AVHs despite adequate clozapine treatment. ${ }^{1,14-17}$ Persistent AVHs are associated with increased violence, hospitalization, risk of suicide, ${ }^{18}$ and low likelihood of future recovery. ${ }^{19,20}$ Despite this, there are few long-term follow-up studies on the subjective experience of AVHs in clozapine-medicated patients. ${ }^{15}$

The aim of this retrospective study was to explore longitudinal changes in AVHs assessed with HPSVQ in patients with schizophrenia who had been treated with clozapine. We also investigated the effects of various factors on long-term change trajectories.

\section{METHODS}

\section{Subjects}

Patients with schizophrenia suffering from AVHs between 
20 and 65 years of age were recruited. The eligible subjects were patients who met the Diagnostic and Statistical Manual of Mental Disorders, Fourth Edition Text Revision criteria of schizophrenia, had been treated with clozapine, and assessed with HPSVQ more than twice during the observation period from March 2012 to February 2016. This study was approved by the Institutional Review Board of Dongguk University International Hospital (\#2016-125).

\section{The HPSVQ}

HPSVQ was developed as a 13-item self-report questionnaire on a 5-point Likert scale to address the subjective effects of AVHs. ${ }^{7}$ The total score of the first 9 items was taken as the severity of subjective distress. The Korean version of HPSVQ were in good agreement with PSYRATS-AHS and the Positive and Negative Syndrome Scale (PANSS)-P3 item, and also showed good test-retest reliability and internal consistency. ${ }^{8}$ In our previous study, two factors of HPSVQ were identified and named as physical and emotional factors, respectively. ${ }^{8}$

\section{Statistical analysis}

Demographic and clinical characteristics were evaluated at baseline. Longitudinal changes of the total and factor scores of HPSVQ were modeled using linear mixed-effect regression. The effect of the covariates on the overall mean of the response variables, and on the slopes of time-dependent changes were evaluated. The right-skewed variables such as duration of treatment (DOT) with clozapine and duration of illness were log-transformed. The significance of the calculated coefficients was tested by the Wald t-test.

The observation periods of the patients were not homogeneous and several patients were lost during the study period. Thus the Cox proportional hazard model was employed to adjust for the censored data. It could estimate the effect of covariates on the proportional hazard ratios. The target event was defined as an at-least 50\% reduction in the total HPSVQ score from the baseline. The first point of measurement when the HPSVQ score was less than half the baseline was regarded as the time to the event for each subject. The appropriateness of the proportional hazard assumption was checked using the goodness-of-fit test based on Schoenfeld residuals. All statistical analyses were performed using R software (ver. 3.2.4: R Project for Statistical Computing, Vienna, Austria). ${ }^{21}$ A pvalue $<0.05$ was considered significant.

\section{RESULTS}

\section{Demographic and clinical characteristics}

Among the 55 subjects originally participated in the study, 11 patients with HPSVQ total scores $\leq 7$ (indicating absent-to- minimal AVHs) ${ }^{8}$ at baseline were excluded. The data of remaining 44 patients were summarized in Table 1 . No significant differences in the demographic and clinical information were detected between the excluded and analyzed patients.

\section{Longitudinal changes in the HPSVQ}

The mean total score of HPSVQ was significantly decreased from $16.2 \pm 5.6$ at baseline to $10.4 \pm 8.6$ at the last observation $(\mathrm{t}=4.441, \mathrm{p}=0.000$ by paired $\mathrm{t}$-test $)$. All items except "interference with life" and two factor scores of HPSVQ decreased significantly during the observation period.

The trajectories of the total and factor scores of HPSVQ were visually inspected. All three scores changed markedly during the first year of the observation period, but the emotional factor did not show such a noticeable change. Formal statistical tests with linear mixed-effect regression also supported such findings. Total score decreased by 9.2 points per year $[\mathrm{t}(263)=$ $-3.60, \mathrm{p}=0.0004]$, the physical factor score by 5.8 points per year $[\mathrm{t}(263)=-3.64, \mathrm{p}=0.0003]$, and the emotional factor score by 3.4 points per year $[\mathrm{t}(263)=-2.476, \mathrm{p}=0.0139]$.

Gender exerted a significant effect on the overall mean of total score $[\mathrm{t}(40)=2.16, \mathrm{p}=0.037]$ and physical factor score $[\mathrm{t}(40)=2.05, \mathrm{p}=0.047]$, but not of the emotional factor score $[\mathrm{t}(40)=1.72, \mathrm{p}=0.094]$. Female patients reported more AVHs and more severe distress than males. And the log-transformed DOT with clozapine had a significant effect on the HPSVQ scores [total score: $\mathrm{t}(40)=2.86, \mathrm{p}=0.0067$, physical factor score: $\mathrm{t}(40)=2.61, \mathrm{p}=0.013$, emotional factor score: $\mathrm{t}(40)=2.39, \mathrm{p}=$ 0.019]. The coefficients indicate that if DOT was doubled then the total, physical and emotional factor scores would increase

Table 1. Demographic and clinical characteristics of the subjects with AVHs

\begin{tabular}{lc}
\hline Subjects (number) & $\mathrm{N}=44$ \\
Age (years) & $36.4 \pm 9.6$ \\
Sex & \\
$\quad$ Male & $24(54.5 \%)$ \\
$\quad$ Female & $20(45.5 \%)$ \\
Duration of Illness (years) & $14.6 \pm 9.2$ \\
$\quad$ (Median) & 13.5 \\
Duration of clozapine trial (years) & $4.6 \pm 5.3$ \\
$\quad$ (Median year) & 1.83 \\
Family history & $32(72.7 \%)$ \\
$\quad$ Negative & $12(27.3 \%)$ \\
$\quad$ Positive & $221.3 \pm 127.3$ \\
Baseline clozapine dose (mg/day) & $492.5 \pm 350.1$ \\
Observation period (days) & 452 \\
$\quad$ (Median days) & \\
\hline AVHs: auditory verbal hallucinations &
\end{tabular}


by $1.09,0.59$, and 0.51 points, respectively.

Gender also had a significant modifying effect on the timedependent changes in total and physical factor scores. It was estimated that the increases (per year) in the total and physical factor scores were 6.48 and 3.70 points higher in female patients than in males, respectively.

\section{Time-to-event analysis using Cox proportional hazard regression}

Only 21 patients reached the pre-defined threshold for the event (47.8\%) during the 1-year observation period, therefore the first quartile (25\%) survival time was calculated (35 days) instead of the median. DOT with clozapine had a significant effect on the hazard ratio of reaching the event $(z=-2.69, p=$ 0.007). The coefficient suggests that doubling the DOT with clozapine decreased the likelihood of reaching the event by $24 \%$. Figure 1 shows the adjusted survival curves for the Cox proportional hazard model. The survival curves of the patients with a longer DOT with clozapine were shifted significantly upwards, suggesting a lower likelihood of reaching the threshold for the event.

\section{DISCUSSION}

We demonstrated that subjective aspects of AVHs evaluated with HPSVQ gradually improved during a 3-year observa- tion period in patients with clozapine treated schizophrenia. Female patients and patients with longer DOT were more resistant to the effect of clozapine. This result indicated the beneficial effect of clozapine on AVHs, ${ }^{14-16}$ particularly on subjective dimension.

Similarly looking item questionnaire can elicit quite different aspects of personal experience according to the method of assessment. ${ }^{22}$ For example, the item "interference with life" of HPSVQ have not signiticantly decreased during the observation period. In our previous study, the item "disruption to life" of PSYRATS-AHS showed a gradual reduction during the 6 months of observations, ${ }^{10,15}$ wherease, the "interference with life" of HPSVQ remained unchanged during the 1 year followup. ${ }^{23}$ Moreover, the present study showed that, unlike the physical factor, the emotional factor was quite resistant to change. These findings are consistent with the previous reports that emotional disruption could not be easily improved despite longterm treatment. ${ }^{24,25}$ These results supported the distinctive value of self-report in AVHs researches.

The time-dependent changes were not even during the observation period; the total and physical factor changed markedly during the first year of observation and reached plateau thereafter, whereas the emotional factor did not change much during the early phase. The likelihood of HPSVQ total score improvement decreased by $24 \%$ with a doubling of DOT with clozapine. This is consistent with previous reports that longer-

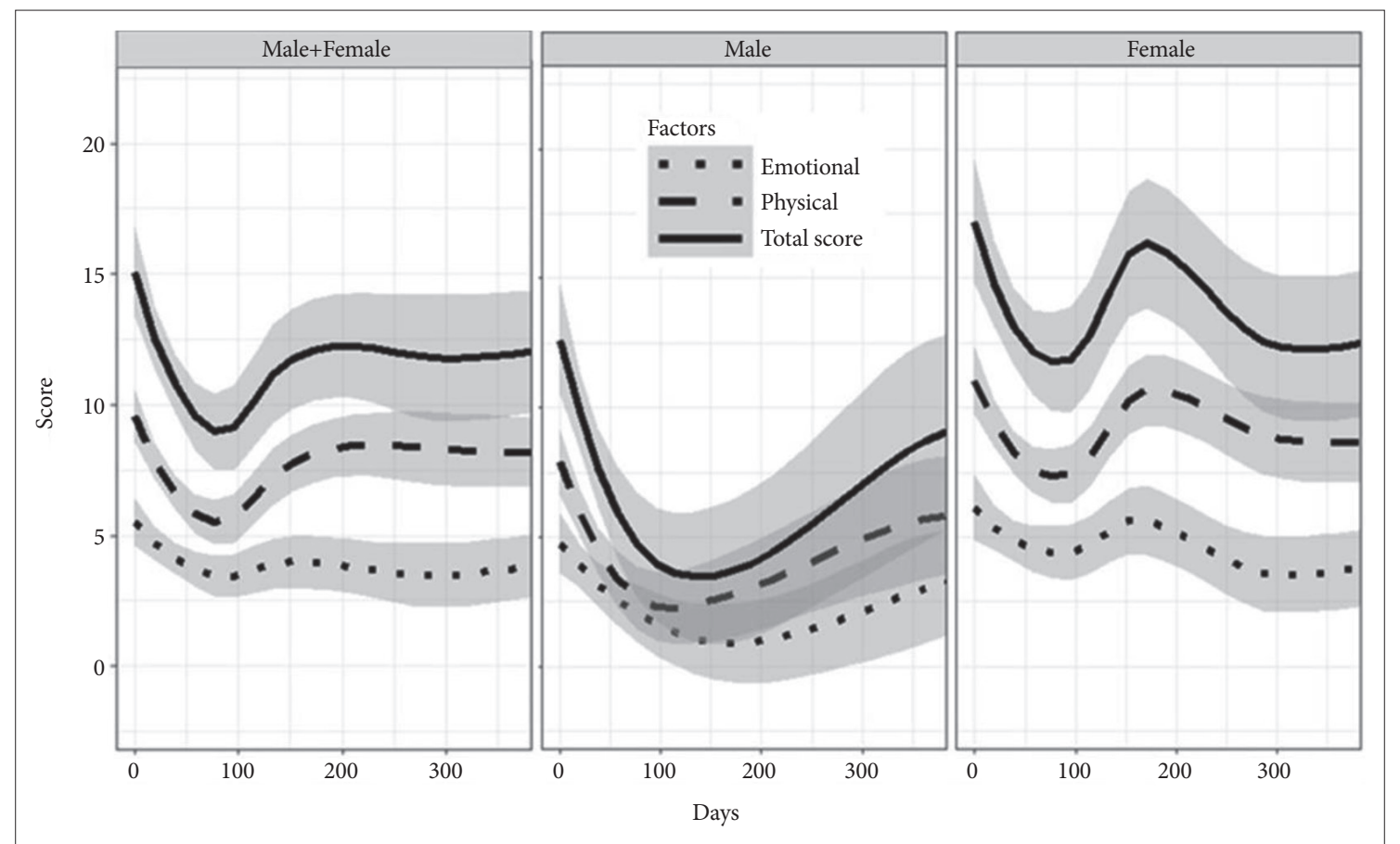

Figure 1. 1-year change trajectories of the total, physical, and emotional factor scores of the HPSVQ. The depicted trajectories were calculated by averaging the individual scores belonging to the same time-period. The smoothed curve was obtained through Loess local regression fitting (Loess stands for locally weighted scatterplot smoothing). HPSVQ: Hamilton Program for Schizophrenia Voices Questionnaire. 
lasting AVHs increased the likelihood of becoming refractory. ${ }^{1,26}$ Taken together, the physical dimention of AVHs seemed to respond earlier but to become more and more resistant, while the emotional dimension seemed to take more time to meaningful improvement.

Several limitations should be mentioned. First, the number of patients was small for tracking long-term course of AVHs, the numbers and intervals of the assessments were inconsistent. Second, the doses of clozapine and the other psychotropics were not controlled during the study; this may have affected the psychotic symptoms of the patients, although only patients treated with clozapine were included.

In conclusion, self-report AVHs in patients with schizophrenia using clozapine decreased markedly in the early phase of observation, fluctuated thereafter, but eventually decreased. Female gender and DOT with clozapine had negative associations with the degree of HPSVQ improvement. Further studies are needed to clarify why the decrease in the emotional factor was less marked and took more time to improve with clozapine treatment.

\section{Acknowledgments}

Dr. Kim YS received grants, research support and honoraria from Janssen and Otsuka.

\section{Conflicts of Interest}

The authors have no potential conflicts of interest to disclose.

\section{Author Contributions}

Conceptualization: Yong Sik Kim, In Won Chung. Data curation: Hee Yeon Jung, Seong Hoon Jeong. Formal analysis: Seong Hoon Jeong. Investigation: Se Hyun Kim, Tak Youn. Methodology: Tak Youn, Hee Yeon Jung. Project administration: In Won Chung, Se Hyun Kim. Resources: Tak Youn, Se Hyun Kim. Software: Seong Hoon Jeong, Hee Yeon Jung. Supervision: Yong Sik Kim. Validation: In Won Chung, Yong Sik Kim. Visualization: In Won Chung, Seong Hoon Jeong. Writing-original draft: In Won Chung. Writing — review \& editing: Seong Hoon Jeong, Yong Sik Kim.

\section{ORCID iDs}

$\begin{array}{ll}\text { Yong Sik Kim } & \text { https://orcid.org/0000-0002-8845-5186 } \\ \text { In Won Chung } & \text { https://orcid.org/0000-0002-7852-7430 }\end{array}$

\section{REFERENCES}

1. González JC, Aguilar EJ, Berenguer V, Leal C, Sanjuan J. Persistent auditory hallucinations. Psychopathology 2006;39:120-125.

2. Schneider K. Clinical Psychopathology. New York: Grune \& Stratton; 1959.

3. Shergill SS, Murray RM, McGuire PK. Auditory hallucinations: a review of psychological treatments. Schizophrenia Res 1998;32:137-150.

4. Haddock G, McCarron J, Tarrier N, Faragher EB. Scales to measure dimensions of hallucinations and delusions: the psychotic symptom rating scales (PSYRATS). Psychol Med 1999;29:879-889.

5. Upthegrove R, Ives J, Broome MR, Caldwell K, Wood SJ, Oyebode F. Auditory verbal hallucinations in first-episode psychosis: a phenomeno- logical investigation. B J Psych Open 2016;2:88-95.

6. Chang JS, Jeong SH, Ahn YM, Kim YS. Multi-dimensional changes in auditory verbal hallucination during antipsychotic treatment of schizophrenic patients. J Korean Neuropsychiatr Assoc 2005;44:65-74.

7. van Lieshout RVJ, Goldberg JO. Quantifying self-reports of auditory verbal hallucinations in persons with psychosis. Can J Behav Sci 2007;39: 73-77.

8. Kim SH, Jung HY, Hwang SS, Chang JS, Kim Y, Ahn YM, et al. The usefulness of a self-report questionnaire measuring auditory verbal hallucinations. Prog Neuropsychopharmacol Biol Psychiatry 2010;34:968973.

9. Spitzer M. The Basis of Psychiatric Diagnosis. In: Sadler JZ, Wiggins OP, Schwartz MA, Editors. Johns Hopkins Series in Psychiatry and Neuroscience. Philosophical Perspectives on Psychiatric Diagnostic Classification Baltimore: Johns Hopkins University Press, 1994, p.163-177.

10. Junginger J, Frame CL. Self-report of the frequency and phenomenology of verbal hallucinations. J Nerv Ment Dis 1985;173:149-155.

11. Correll CU, Kishimoto T, Nielsen J, Kane JM. Quantifying clinical relevance in the treatment of schizophrenia. Clin Ther 2011;33:B16-B39.

12. Hazell CM, Hayward M, Cavanagh K, Jones AM, Strauss C. Guided selfhelp cognitive-behaviour Intervention for VoicEs (GiVE): Results from a pilot randomised controlled trial in a transdiagnostic sample. Schizophr Res 2018;195:441-447.

13. Ratcliff K, Farhall J, Shawyer F. Auditory hallucinations: a review of assessment tools. Clin Psychol Psychother 2011;18:524-534.

14. Pandarakalam JP. Pharmacolobical and non-pharmacological interventions for persistent auditory hallucinations in schizophrenia. Br J Med Pract 2016;9:a914.

15. Sommer IEC, Slotema CW, Daskalakis ZJ, Derks EM, Blom JD, van der Gaag M. The treatment of hallucinations in schizophrenia spectrum disorders. Schizophr Bull 2012;38:704-714.

16. Li Q, Xiang YT, Su YA, Shu L, Yu X, Correll CU, et al. Clozapine in schizophrenia and its association with treatment satisfaction and quality of life: Findings of the three national surveys on use of psychotropic medications in China (2002-2012). Schizophr Res 2015;168:523-529.

17. Nasrallah HA. Treatment-resistant schizophrenia. Curr Psychiatry 2014; 13:24-25.

18. Hor K, Taylor M. Suicide and schizophrenia: a systematic review of rates and risk factors. J Psychopharmacol 2010;24(4 Suppl):81-90.

19. Goghari VM, Harrow M, Grossman LS, Rosen C. A 20-year multi-follow-up of hallucinations in schizophrenia, other psychotic, and mood disorders. Psychol Med 2013;43:1151-1160.

20. Koops S, Blom JD, Bouachmir O, Slot MI, Neggers B, Sommer IE. Treating auditory hallucinations with transcranial direct current stimulation in a double-blind, randomized trial. Schizophr Res 2018;201:329-336.

21. R Core Team. R: A Language and Environment for Statistical Computing. Vienna, Austria: R Foundation for Statistical Computing,; 2016.

22. Hayhurst KP, Massie JA, Dunn G, Lewis SW, Drake RJ. Validity of subjective versus objective quality of life assessment in people with schizophrenia. BMC Psychiatry 2014;14:365.

23. Kim SH, Park HG, Jeong SH, Kang UG, Ahn YM, Kim YS. Electroconvulsive seizure alters the expression and daily oscillation of circadian genes in the rat frontal cortex. Psychiatry Investig 2018;15:717-726.

24. Miller LJ. Qualitative Changes in Hallucinations. Am J Psychiatry 1996; 153: 265-267.

25. Schneider SD, Jelinek L, Lincoln TM, Moritz S. What happened to the voices? A fine-grained analysis of how hallucinations and delusions change under psychiatric treatment. Psychiatry Res 2011;188:13-17.

26. Drake RJ, Dunn G, Tarrier N, Haddock G, Haley C, Lewis S. The evolution of symptoms in the early course of nonaffective psychosis. Schizophr Res 2003;63:171-179. 